\title{
La diagnostica dei micobatteri in Italia*
}

\author{
Claudio Piersimoni \\ Comitato AMCLI per lo Studio dei Micobatteri (CoSMic)
}

* II presente articolo è tratto da una relazione tenuta al XXXIII Congresso Nazionale AMCLI (Padova 8- I I giugno 2004) successivamente pubblicata sotto forma di "Research Note" su Clin. Microbiol. Infect. 2004; I 0: I0 I4- I0I 7.

La tubercolosi può essere considerata una emergenza planetaria, dal momento che si registrano ogni anno circa 8 milioni di nuovi casi e oltre due milioni di decessi. La prevalenza della malattia nei paesi industrializzati è fortunatamente bassa anche se si registrano epidemie causate da ceppi di Mycobacterium tuberculosis complex (MTB) multi resistenti all'interno di ospedali, prigioni e ricoveri per senza tetto che hanno coinvolto prevalentemente soggetti HIV-positivi ed immigrati. Dal momento che la malattia si trasmette da persona a persona, è assolutamente necessario che $\mathrm{i}$ casi siano diagnosticati il più rapidamente possibile ed altrettanto rapidamente messi in isolamento respiratorio ed adeguatamente trattati.

I laboratori di microbiologia giocano un ruolo chiave nel contrastare il ritorno della tubercolosi. Per tale motivo, viene oggi considerata improcrastinabile l'adozione di quei metodi in grado di fornire accurati isolamento, identificazione ed antibiogramma (DST) del MTB riducendo al massimo i tempi di refertazione $(7,11)$.

Al fine di verificare il rispetto delle indicazioni diagnostiche sopra riportate da parte dei laboratori Ospedalieri Italiani, il Comitato per lo Studio dei Micobatteri (CoSMic) ha messo a punto un questionario nel quale venivano richieste informazioni riguardanti i metodi usati per microscopia, decontaminazione, coltura, identificazione, DST, tempi di refertazione e dotazioni di sicurezza. Ai laboratori è stato anche chiesto di fornire l'entità dei carichi di lavoro in termini di numero di campioni processati, ceppi di MTB identificati, numero di DST eseguiti, ceppi inviati ad altri laboratori e l'eventuale utilizzo dei test di amplificazione diretta da campione.

L'indagine è stata condotta nel periodo 19992001 e l'attività rilevata ha coperto un intervallo di 12 mesi (1 gennaio-31 dicembre). I dati sono stati inviati dalle delegazioni regionali al CoSMic e successivamente elaborati usando i software Epi Info 5.0 e Microsoft Excel.

È stato possibile raccogliere informazioni da 355 laboratori ospedalieri. In tutti viene eseguita la ricerca microscopica per bacilli alcool-acido resistenti (BAR), 298 (89.3\%) ricercano i micobatteri mediante coltura primaria, $133(37.5 \%)$ sono in grado di identificare MTB, 83 (23.4\%) eseguono il DST sui ceppi identificati e 73 (20.6\%) ricerca- no MTB direttamente da campione mediante amplificazione. Naturalmente la maggior parte dei laboratori più grandi eseguono tutte le suddette procedure. Passando ad esaminare i dati più in dettaglio, nel periodo studiato sono stati eseguiti complessivamente 242.381 esami microscopici e 218.991 esami colturali. Relativamente ai carichi di lavoro, 179 laboratori $(50.4 \%)$ processano non più di 500 campioni l'anno, $105(29.6 \%)$ non più di 3.000 a solamente $14(3.9 \%)$ trattano più di 3.000 campioni l'anno. Dei 57 laboratori (16.1\%) che non eseguono colture, 35 (9.9\%) inviano gli isolati a laboratori più grandi, mentre in $22(6.2 \%)$ le colture non vengono eseguite affatto. Sebbene questo trend è abbastanza omogeneo in tutto il paese, esso sembra più pronunciato nelle regioni del centro-sud. In totale sono stati identificati 10.045 ceppi di MTB e di questi 5.649 sono stati testati nei confronti dei farmaci antitubercolari di prima scelta. Il numero totale dei test di amplificazione diretta (DATs) eseguiti durante il periodo di studio è ammontato a 22.270 , di cui 2.723 $(11.9 \%)$ positivi. La ricerca microscopica prevede l'utilizzo della colorazione di Ziehl-Neelsen nell' $81.4 \%$ dei partecipanti, dei fluorocromi nel $14.4 \%$ e della colorazione a freddo di Kynioun nel restante 4.2\%. Nei laboratori (298 su 355) che praticano la coltura dei micobatteri, si utilizzano diversi metodi di decontaminazione: il metodo di riferimento $\mathrm{N}$-acetyl cysteina idrossido di sodio (NALC-NaOH) è in uso in 166 laboratori (55.7\%), laddove nei restanti 132 (44.3\%) si adoperano metodiche che impiegano differenti concentrazioni di $\mathrm{NaOH}$, oppure diversi agenti decontaminanti e sostanze fluidificanti. La coltura primaria viene eseguita dal $46.7 \%$ dei partecipanti utilizzando la combinazione di un sistema liquido non radiometrico e di terreno solido, dal $6 \%$ mediante sistema radiometrico e terreno solido, mentre nel $47.3 \%$ dei casi viene impiegato il solo terreno solido (Tabella 1). Fra i sistemi liquidi non radiometrici i più usati sono quelli automatizzati (BACTEC 9000 MB, BACTEC MGIT 960 [Becton Dickinson Biosciences, Sparks MD], MB/BacT Alert [Organon Teknika, Durham, NC], and ESP II [ESP Culture System II, Trek Diagnostics, Westlake, Ohio]), seguiti da quelli manuali (MGIT, Septi-Check AFB [Becton Dickinson Biosciences, Sparks MD], e MB 
Redox [Biotest AG, Dreieich, Germany]), laddove fra i terreni solidi quelli di gran lunga più impiegati sono il Löwenstein-Jensen (L-J) e lo IUTM. Tutti i laboratori più grandi associano un sistema liquido radiometrico o non radiometrico con il terreno di L-J per la coltura routinaria dei micobatteri. Fra i laboratori che eseguono la coltura, $133(44.7 \%)$ hanno fornito informazioni relative ai metodi di identificazione del Mycobacterium tuberculosis a livello di specie o di complesso (Tabella 1). A tal riguardo, 28 laboratori impiegano test biochimici, 85 usano metodi molecolari con o senza amplificazione del target (i test di amplificazione diretta da campione possono essere usati per identificare il MTB da colture in fase precoce di crescita), 20 utilizzano il NAP (p-nitro$\alpha$-acetylamino- $\beta$-hydroxypropiophenone) test radiometrico, mentre la cromatografia liquida (HPLC) non viene utilizzata a questo scopo e trova impiego in due laboratori di riferimento esclusivamente per la identificazione dei NTM. Tra i 133 laboratori che identificano MTB, 83 (27.5\% del totale dei laboratori) eseguono il test di sensibilità (DST).

Tabella I. Metodi in uso presso i laboratori censiti.

METODI IN USO N. (\%) DEI LABORATORI

\begin{tabular}{lc}
\hline Ziehl-Neelsen & $289(8 \mathrm{I} .4)$ \\
\hline Fluorocromi & $5 \mathrm{I}(14.4)$ \\
\hline Kinyoun & $15(4.2)$
\end{tabular}

Kinyoun

\begin{tabular}{lc}
\hline Terreno solido & I4I (47.3) \\
\hline Terreno solido e radiometrico & I8 (6.0) \\
\hline Terreno solido e non-radiometrico & $139(46.7)$ \\
\hline Identificazione di MTB & \\
\hline DNA probes e/o DATs & \\
\hline BACTEC NAP & $20(28.6)$ \\
\hline Test biochimici & $28(6.7)$ \\
\hline HPLC & \\
\hline Non eseguita & $0(0.0)$ \\
\hline
\end{tabular}

Test di sensibilità ai farmaci per MTB

Metodo proporzionale in terreno all'uovo 27 (9.1)

Metodo proporzionale in agar I (0.3)

Metodo Radiometrico 34 (I I.4)

Terreni liquidi non-radiometrici $20(6.7)$

${ }^{\mathrm{a} D A T s}$ : test di amplificazione diretta; ${ }^{\mathrm{b} H P L C}$ : high performance liquid chromatography.

Dei 133 laboratori che completano la identificazione di MTB, 83 (27.5\% di tutti i laboratori censiti) eseguono il test di sensibilità sui ceppi identificati a partire da una coltura pura del microrganismo (metodo indiretto) adottando il metodo delle proporzioni. Ventisette laboratori su 83 (33.2\%) usano terreni all'uovo come il L-J o lo IUTM, 34 $(41.3 \%)$ usano il metodo radiometrico, 20 (24.3\%) ricorrono ai sistemi liquidi non radiometrici men- tre un solo laboratorio esegue il DST secondo il metodo di riferimento NCCLS (Middlebrook $7 \mathrm{H} 10$ o 7H11). La maggior parte dei laboratori testano streptomicina, isoniazide, rifampicina ed etambutolo, mentre la pirazinamide è testata solo dagli utilizzatori del sistema radiometrico. I laboratori sono stati indagati anche relativamente all'uso dei test diretti di amplificazione specificando se essi fossero commerciali o "homemade". Nel nostro paese, il $20 \%$ dei laboratori (73/355) fa regolare uso di DATs, ma solo 1'1,4\% (6/355) prepara i test "in casa". In media, i risultati dell'esame microscopico sono disponibili dopo 3 giorni dal ricevimento del campione e questo tempo medio cresce man mano che si scende dal nord verso il sud $(2.6,2.9$ e 3.5 giorni per nord, centro e sud rispettivamente).

Per quanto attiene al rispetto delle norme di sicurezza (2, 4), $107(33.7 \%)$ dei 317 laboratori rispondenti dispone di una stanza di isolamento o più semplicemente di una stanza dedicata ed in 4 $(1.3 \%)$ è in funzione un sistema di ventilazione a pressione negativa. La cabine di sicurezza di classe II (BSC) sono disponibili in 269 (84.8\%) laboratori, mentre una centrifuga dedicata alle procedure di micobatteriologia viene riportata in 117 (36.9\%) e solo 146 laboratori (46.1\%) dichiarano di possedere chiusure di sicurezza anti-aerosol applicate ai cestelli delle centrifughe.

In sintesi, la nostra indagine ha mostrato che solo il $14.4 \%$ dei laboratori usa la tecnica dei fluorocromi per la microscopia e che poco più della metà dei laboratori $(55.7 \%)$ utilizza al tecnica standard NALC-NaOH per la decontaminazione dei campioni. Le metodiche di decontaminazione alternative pur essendo talvolta decisamente fantasiose, hanno il difetto di essere lesive per i micobatteri riducendo in tal modo la quantità di micobatteri vitali presenti nel campione.

Il $52.7 \%$ dei laboratori censiti pratica la coltura primaria dei micobatteri utilizzando la combinazione di terreno solido e terreno liquido, mentre per la identificazione e il DST il 35.3 ed $18.4 \%$ rispettivamente impiegano i metodi rapidi raccomandati dal CDC. Tra i sistemi liquidi non radiometrici, risultano prevalenti quelli che negli USA non hanno ottenuto la licenza della prestigiosa Food and Drug Administration (FDA). Lo stesso vale per le metodiche di amplificazione che solo nell' $11.3 \%$ risultano essere appannaggio di sistemi "FDA-approved".

Una raccomandazione ufficiale della American Thoracic Society [ATS](1) suggerisce che al fine di mantenere una adeguata competenza nella microscopia, coltura ed identificazione dei micobatteri è necessario leggere almeno $10-15$ vetrini a settimana e processare non meno do 20 coltura 
a settimana. In base ai dati raccolti, meno del $20 \%$ $(19.7 \%)$ dei laboratori censiti rientra nei parametri qualitativi sopra riportati. Questi dati da un lato non possono non suscitare imbarazzo, dall'altro indicano la necessità non più procrastinabile affinché la microbiologia dei micobatteri sia concentrata in pochi laboratori specializzati. In Italia, questa strategia soddisfa i parametri della costoefficacia e ben si sposa con la configurazione del Sistema Sanitario Nazionale e le attuali politiche di decentramento sanitario (10).

Le cose non vanno meglio nel campo della biosicurezza. Infatti, 2/3 dei laboratori non sono dotati di una stanza dedicata alle procedure di micobatteriologia, la ventilazione forzata a pressione negativa è praticamente assente e per finire circa il 15\% dei laboratori non possiede una cappa a flusso laminare di classe II ed il 50\% degli stessi è priva di una centrifuga con protezione antiaerosol. Lo studio non è privo di "bias": primo fra tutti il fatto di non essere riuscito a censire tutti i laboratori ospedalieri pubblici e di non aver considerato quelli privati. Ciononostante, la popolazione studiata può essere considerate rappresentativa della realtà nazionale per numero e dimensione dei laboratori. Una precedente indagine (5) condotta su 99 laboratori situati in ospedali dotati di Divisioni di Malattie Infettive aveva mostrato che il $57.6 \%$ non identificava i ceppi di MTB ed il $42.6 \%$ non eseguiva il DST. La situazione della biosicurezza non era meno fosca: il $15.1 \%$ dei laboratori non possedeva cappe a flusso laminare di classe II e nel $28 \%$ dei casi le procedure di micobateriologia non prevedevano l'uso di una centrifuga protetta.

In Italia, per rendere la diagnosi di laboratorio della $\mathrm{TB}$ più rapida per il paziente e più sicura per gli addetti ai lavori è assolutamente indispensabile porre rimedio alla frammentazione della diagnostica indirizzandola per intero presso un numero limitato di laboratori specializzati scelti sulla base del carico di lavoro e della specifica competenza professionale.

Inoltre, numerosi studi $(3,6,8,12,13)$ hanno dimostrato che importanti fattori quali le caratteristiche strutturali del laboratorio, le dotazioni tecniche, la formazione del personale e soprattutto il rapporto fra personale e carichi di lavoro possono influenzare considerevolmente la qualità della diagnostica erogata anche in strutture altamente specializzate come i laboratori di riferimento. In altri termini, ciò significa che non serve creare una rete di laboratori di riferimento se poi non si opera per dotarli di adeguate risorse. Nel nostro paese un modello di razionalizzazione dei laboratori di micobatteriologia sulla scorta di quello proposto dalla ATS (1) è stato introdotto nel 1998 dal decreto legislativo 31 marzo 1998, n. 112 (9), ma soltanto pochissime regioni hanno tentato di adeguarsi al dettato di legge fornendo adeguati piani di razionalizzazione e risorse per la loro attuazione. Oltre 10 anni fa Tenover et al (11) concludendo un articolo che viene oggi considerato storico per i micobatteriologi, pose la famosa domanda: "è pronto il tuo laboratorio (ad affrontare il ritorno della TB)"? Dispiace dover concludere che in Italia nulla è stato fatto dalle Istituzioni (a tutti i livelli) affinché gli operatori del settore possano legittimamente rispondere: "Sì !"

\section{Ringraziamenti}

Gli autori ringraziano i seguenti colleghi per la assistenza gentilmente concessa nel fornire i dati: Maria G. Mazzarello (Ovada), Panajota Troupioti (Sondalo), Giorgio Mucignat (Pordenone), Gianna De Fina (Bolzano), Iole Caola (Trento), Maria L. Moro (Bologna), Giovanni Sbaraglia (Perugia), Maria G. Proietti (Terni), Patrizia Chiaradonna (Roma), Paolo Fazii (Pescara), Marina Bucci (Larino), Marco Liguori (Cagliari), Giuseppe Miragliotta (Bari), Marco Conte (Napoli) e Paolina Cavalcanti (Cosenza).

\section{BIBLIOGRAFIA}

1. American Thoracic Society. Level of laboratory services for mycobacterial diseases. Am Rev Respir Dis 1983; 128: 213.

2. Barenfanger J. Making your lab safe against multidrug-resistant Mycobacterium tuberculosis. Clin Microbiol News 1993; 15: 76-80.

3. Bird BR, Denniston MD, Huebner RE, Good RC. Changing practise in mycobacteriology: a follow-up survey of state and territorial public health laboratories. J Clin Microbiol 1996; 34: 554-9.

4. $\mathrm{CDC} / \mathrm{NIH}$. Proposed guidelines for goals for working safely with Mycobacterium tuberculosis in clinical, public health and research laboratories. U.S. Department of Health and Human Services, Centers for Disease Control and Prevention, 1997.

5. Chiaradonna P, Girardi E, Spanò A, Tronci M. Risultati dell'indagine conoscitiva nazionale sulla diagnostica delle infezioni da micobatteri negli ospedali sede di reparti di Malattie Infettive. Microbiologia Medica 1996; 11: 59-62.

6. Drobniewski FA, Watt B, Smith EG, Magee JM, Williams R, Holder J, Ostrowski J. A national audit of the laboratory diagnosis of tuberculosis and other mycobacterial diseases in the United Kingdom. J Clin Pathol 1999; 52: 334-7.

7. Hinman AR, Hughes JM, Snider DE, Cohen ML. meeting the challenge of multidrug-resistant tuberculosis: summary of a conference. Morbid Mortal Weekly 1992; Rep. 41 (no. RR-11): 51-7.

8. Huebner RE, Goods RC, Tokars JI. Current practices in mycobacteriology: results of a survey of state public health laboratories. J Clin Microbiol 1993; 31: $771-5$. 
9. Linee-guida per il controllo della malattia tubercolare, su proposta del Ministero della Sanità, ai sensi dell'art. 115, comma 1, lettera b), del decreto legislativo 31 marzo 1998, n. 112.

10. Remuzzi G. Country profile: Italy. Lancet 1996; 348 : 167-75.

11. Tenover FC, Crawford JT, Huebner RE, Geiter LJ, Horsburgh CR, Jr. and RC Good. The resurgence of tuberculosis: is your laboratory ready? J Clin Microbiol 1993; 31: 767-70.

12. Tokars JI, Rudnick JR, Kroc K, et al. U.S. hospitals mycobacteriology laboratories: status and comparison with state public health department laboratories. J Clin Microbiol 1996; 34: 680-5.

13. Woods GL, Witebsky FG. Mycobacterial testing in clinical laboratories that participate in the college of American pathologists' mycobacteriology E survey: results of a 1993 questionnaire. J Clin Microbiol 1995; 33: 407-12.

\section{Claudio Piersimoni}

Sezione di Microbiologia, Laboratorio di Patologia Clinica Dipartimento di Patologia ed Analisi Azienda Ospedaliera "Ospedali Riuniti" Via Conca 71, I-60020, Ancona Tel.: 071-596.3049; Fax: 071-596.4184 E-mail: piersim@tin.it 